Research Article

\title{
A Novel ULA-Difference-Coarray-Based DOA Estimation Method for General Coherent Signals
}

\author{
Zhang Chen $(\mathbb{D}$, Hao Wu $(\mathbb{D}$, and Yongxiang Liu $(\mathbb{D}$ \\ The Sixty-Third Institute, National University of Defense Technology, Nanjing 210007, China \\ Correspondence should be addressed to Yongxiang Liu; lyx63s@163.com
}

Received 20 August 2020; Revised 3 October 2020; Accepted 22 October 2020; Published 3 November 2020

Academic Editor: Wang Zheng

Copyright (C) 2020 Zhang Chen et al. This is an open access article distributed under the Creative Commons Attribution License, which permits unrestricted use, distribution, and reproduction in any medium, provided the original work is properly cited.

In this article, a difference-coarray-based direction of arrival (DOA) method is introduced, which utilizes the uniform linear array (ULA) in a novel fashion to address the problem of DOA estimation for coherent signals. Inspired by the coarray-based estimators employed in cases of sparse arrays, we convert the sample covariance matrix of the observed signals into the difference coarray domain and process the signals using a spatial smoothing technique. The proposed method exhibits good accuracy and robustness in both the uncorrelated and coherent cases. Numerical simulations verify that the ULA difference coarray- (UDC-) based method can achieve good DOA estimation accuracy even when the SNR is very low. In addition, the UDC-based method is insensitive to the number of snapshots. Under extremely challenging conditions, the proposed UDC-ES-DOA method is preferred because of its outstanding robustness, while the UDC-MUSIC method is suitable for most moderate cases of lower complexity. Due to its demonstrated advantages, the proposed method is a promising and competitive solution for practical DOA estimation, especially for low-SNR or snapshot-limited applications.

\section{Introduction}

Antenna-array-based direction of arrival (DOA) estimation algorithms have been widely used in radar, navigation, measurement, and control systems. Various theories and techniques have been developed for array signal processing related to DOA estimation [1]. Uniform linear arrays (ULAs) are often considered in both theoretical research and engineering applications due to their symmetrical and simple array geometries. In this context, subspace-based methods are widely used, including multiple signal classification (MUSIC) [2] and the estimation of signal parameters via rotation invariance techniques (ESPRIT) [3]. In some practical scenarios, such as indoor environments, the signals may be highly correlated (coherent) due to multipath propagation. In this case, the signal covariance matrix is no longer nonsingular, and as a result, the performance of the abovementioned subspace-based DOA algorithms will deteriorate dramatically.

To address this problem, the most common method is to pretreat the observed signals using a spatial smoothing (SS) scheme to recover the reduced rank of the covariance matrix [4]. To cope with K coherent source signals received by a ULA, the forward spatial smoothing (FSS) method requires at least $2 \mathrm{~K}$ sensors, while the forward-backward spatial smoothing (FBSS) method requires $3 \mathrm{~K} / 2$ sensors [5]. Compared to the uncorrelated case, SS approaches realize the decorrelation of coherent signals at the cost of the number of DOFs, and these approaches often lead to worse DOA estimation due to the reduction of the array aperture [6]. Although SS-based approaches are simple and easy to implement, their accuracy and robustness are not satisfactory since the estimation results are sensitive to the SNR and the number of snapshots.

In [7], the Improved MUSIC algorithm was introduced, which enables accurate DOA estimation by reconstructing the covariance matrix of the received signals. In [8], another algorithm, named ES-DOA, was proposed based on an investigation of Improved MUSIC. The ES-DOA algorithm can achieve high and robust performance by making use of both the signal subspace and noise subspace characteristics. The main drawback of both Improved MUSIC and ES-DOA 
algorithms is that they are valid only if there is just one coherent signal. An ESPRIT-like algorithm is proposed to solve the coherent problem by eliminating the rank loss of the spatial covariance matrix with a reconstructed Toeplitz matrix [9-11]. In [12], an MSE-based regularization method is proposed to detect the number of coherent signals and estimate the DOAs. Based on the KR subspace approach, Masaaki et al. proposed the Kronecker-MUSIC method [13], which can cope with $N-1$ coherent source signals by means of $N$ sensors. However, the source signals are restricted to being quasistationary, thus limiting the widespread application of this method. In addition, there are some methods that are developed based on the spatial differencing technique to address the DOA estimation problem of the mixed uncorrelated and coherent sources in multipath environment [14-16]. Compressive sensing- (CS-) based approaches, such as the $l_{1}$-SVD algorithm [17], the JLZA-DOA algorithm [18], and the SBL-DOA algorithm [19], were introduced to identify the DOAs. The CS-based methods are very attractive in that they can cope with the coherent signals with different topologies of the antenna arrays and identify the number of sources automatically [20-22]. Nevertheless, there are basis mismatch problem and heavy computational complexity drawbacks for the CS-based DOA methods.

Recently, approaches based on sparse arrays, such as coprime arrays [23] and nested arrays [24], have been suggested. It is possible to achieve $O\left(N^{2}\right)$ DOFs using $O(N)$ sensors based on a nested array, which is formed by combining two or more ULAs with successively increasing spacing. A number of follow-up methods have been developed to further increase the number of DOFs by modifying the array configurations [25-28]. The difference coarray concept is the foundation of these sparse-arraybased approaches [29]. However, for the existing sparse array estimators, SS schemes cannot be utilized to cope with coherent source signals [30].

Inspired by the coarray-based estimators employed in cases of sparse arrays, we convert the sample covariance matrix of the signals observed by a ULA into the difference coarray domain and pretreat the signals using an SS method. Since the difference coarray of a ULA is another virtual ULA, we can obtain almost twice the original number of DOFs. Furthermore, we utilize the invariance of the ULA shift to handle coherent source signals because the original and virtual arrays both have ULA structures.

The major contributions of this paper include the following:

A novel ULA difference-coarray-based strategy is presented to address the general coherent signal DOA estimate issue. This approach can achieve good accuracy and robustness of DOA estimation in both the uncorrelated and coherent cases.

An enhanced method named UDC-ES-DOA is discussed to solve the DOA estimate problem under extremely challenging conditions. This method can achieve high accuracy with limited snapshots and demonstrates significant superiority in low-SNR scenarios. It is favorable for practical applications.
In addition, numerical simulations are conducted to evaluate the validity of the proposed method. The results show the superiority of the proposed method to the conventional approaches; meanwhile, it can achieve similar performance as the CS-based methods with significantly less computational complexity.

The remainder of the paper is organized as follows. Section 2 introduces the signal model and the conventional methods used in this research. The proposed method is introduced in Section 3. The details of the numerical simulations are discussed in Section 4. Concluding remarks are given in Section 5.

Notations: vectors and matrices are denoted by and lower-case letters $(\mathrm{k})$ and boldface capital letters $(\mathbf{k})$, respectively. Diag $\{\bullet\}$ is a diagonal matrix operator, and vec $(\bullet)$ denotes an operator that returns the vectorization result of a matrix. (_) $)^{\mathrm{T}},\left({ }_{-}\right)^{\mathrm{H}}$, and (_) * stand for transpose, conjugate transpose, and complex conjugate, respectively. $\odot$ depicts the $\mathrm{KR}$ product, and $\otimes$ denotes the Kronecker product.

\section{Preliminaries}

To facilitate the presentation of the proposed method, we shall briefly present some preliminaries regarding the signal model, the FBSS method, difference-coarray-based DOA estimation, and the ES-DOA algorithm.

2.1. Signal Model. Suppose that there are $K(K<N)$ narrowband plane waves impinging on an $N$-element ULA with the source matrix $s(t)=\left[s_{1}(t), s_{2}(t), \ldots, s_{K}(t)\right]^{T}$. Hence, the observed signal is modeled as

$$
\mathbf{x}(t)=\left[x_{1}(t), x_{2}(t), \ldots, x_{N}(t)\right]^{\mathrm{T}}=\mathbf{A s}(t)+\mathbf{n}(t),
$$

where $\mathbf{A}=\left[\mathbf{a}\left(\theta_{1}\right), \mathbf{a}\left(\theta_{2}\right), \ldots, \mathbf{a}\left(\theta_{K}\right)\right] \in \mathbb{C}^{N \times K}$ is the array manifold matrix with Vandermonde-structured distinct columns $(N>K)$ and is of rank $K$, whereas $\mathbf{n}(\mathrm{t}) \in \mathbb{C}^{N}$ represents a white Gaussian noise vector and is not correlated with the sources. The steering vector of the array can be expressed as

$$
\mathbf{a}\left(\theta_{i}\right)=\left[1, e^{-j(2 \pi / \lambda) d \sin \left(\theta_{i}\right)}, \ldots, e^{-j(2 \pi / \lambda)(N-1) d \sin \left(\theta_{i}\right)}\right]^{\mathrm{T}}
$$

where $\theta_{i} \in(-\pi / 2, \pi / 2)$ denotes the DOA of source $i, d$ is the interval of the array, and $\lambda$ is the signal wavelength. If the signal and noise components are stationary, zero-mean, uncorrelated random processes, then the covariance matrix of the received signals can be defined as

$$
\mathbf{R}=E\left[\mathbf{x}(t) \mathbf{x}(t)^{\mathbf{H}}\right]=\mathbf{A} \mathbf{P} \mathbf{A}^{\mathbf{H}}+\boldsymbol{\sigma}_{\mathbf{n}}^{2} \mathbf{I},
$$

where $\mathbf{P}$ is the source autocorrelation matrix of $\mathrm{S}(\mathrm{t}), \sigma_{\mathbf{n}}^{2}$ denotes the noise power, and I denotes the identity matrix. The source covariance matrix $\mathbf{R}$ is a full-rank matrix as long as the incident signals are uncorrelated. The real value of the sample covariance matrix $\mathrm{R}$ is not able to be obtained in practice; instead, the maximum-likelihood estimate of the sample covariance matrix $\widehat{\mathbf{R}}$ is obtained from $M$ snapshots: 


$$
\widehat{\mathbf{R}}=\frac{1}{M} \sum_{i=1}^{M} \mathbf{x}\left(t_{i}\right) \mathbf{x}\left(t_{i}\right)^{\mathbf{H}},
$$

where $M$ is the number of snapshots and $i$ is an index representing different snapshots.

2.2. Forward-Backward Spatial Smoothing. As mentioned above, if the received signals are coherent, then conventional subspace-based algorithms cannot yield accurate DOA estimates because the source autocorrelation matrix $\mathrm{P}$ is not of full rank. By utilizing the invariance of the ULA shift, a spatial smoothing scheme can be used to address this problem.

The basic principle of an SS algorithm is to divide the original ULA into $L$ overlapping uniform subarrays and introduce phase shifts between these subarrays. There are $N-L+1$ sensors in each subarray. The FBSS covariance matrix is given by

$$
\begin{aligned}
R_{\mathrm{FB}} & =\frac{1}{2 L} \sum_{i=1}^{L} F_{i}\left(\mathbf{R}+J \mathbf{R}^{*} J\right) F_{i}^{\mathrm{T}}, \\
\mathbf{F}_{i} & =\left[\mathbf{0}_{(N-L+1) \times(i-1)}\left|\mathbf{I}_{(N-L+1)}\right| \mathbf{0}_{(N-L+1) \times(L-i)}\right],
\end{aligned}
$$

where $\mathbf{J}$ denotes the exchange matrix, with ones on its antidiagonal and zeros elsewhere.

It has been proven that when the number of sensors in the ULA is more than $3 \mathrm{~K} / 2$, the DOA estimates for all coherent signals are available by using the FBSS scheme [5]. However, the SS process significantly reduces the effective array aperture, which often results in a deterioration of the resolution performance.

2.3. Difference-Coarray-Based DOA Method. For sparse linear arrays, such as nested arrays and coprime arrays, the number of DOFs can be significantly increased by exploiting the coarray structure. The difference set of a coarray is based on the KR subspace of the physical array manifold. The KR product has the following important property [31].

Property 1. Let $\mathbf{A} \in \mathbb{C}^{n \times k}, \mathbf{B} \in \mathbb{C}^{n \times k}$, and $\mathbf{d} \in \mathbb{C}^{k}$. In addition, let $\mathbf{D}=\operatorname{Diag}(\mathbf{d})$. Then,

$$
\operatorname{vec}\left(\mathbf{A D B}^{\mathbf{H}}\right)=\left(\mathbf{B}^{*} \odot \mathbf{A}\right) \mathbf{d} .
$$

Under the assumption that all the incident signals are uncorrelated, from (3) and (7), we can obtain the vectorized $\widehat{\mathbf{R}}$ as follows:

$$
\mathbf{r}=\operatorname{vec}(\widehat{\mathbf{R}})=\left(\mathbf{A}^{*} \odot \mathbf{A}\right) \mathbf{p}+\sigma_{n}^{2} \operatorname{vec}(\mathbf{I}),
$$

where $\left[\mathbf{p}=\sigma_{1}^{2}, \sigma_{2}^{2}, \ldots, \sigma_{K}^{2}\right]^{\mathrm{T}}$ and $\mathrm{I}=\left[e_{1}^{2}, e_{2}^{2}, \ldots, e_{K}^{2}\right]^{\mathrm{T}}$, with $\mathrm{e}_{i}$ being a column vector consisting of 1 in the $i$-th position and zeros elsewhere. The new array manifold has dimensions of $N^{2} \times D$.

A coarray with a filled ULA structure that is centered at the origin can be obtained after the sorting and replacement of repeated rows. For a ULA, $\mathbf{A} * \odot \mathbf{A}$ is similar to a
Vandermonde matrix with $(2 N-1)$ distinct rows. The observation vector of the virtual ULA is given by

$$
\mathbf{z}=\mathbf{W r}=\mathbf{A}_{v} \mathbf{p}+\sigma_{n}^{2} \mathbf{W}_{i},
$$

where $\mathbf{W}$ is the coarray selection matrix and $\mathbf{A}_{v}$ denotes the $\left(2 N_{v}+1\right) \times \mathrm{K}$ steering matrix of the $\left(2 N_{v}+1\right)$-element virtual ULA.

Since the difference coarray is a filled ULA, intuitively, it can be applied for DOA estimation. However, the equivalent source signal vector $\mathbf{p}$ consists of the powers of the actual source signals and behaves like the coherent sources in (6).

SS processing can be employed to solve the rank reconstruction problem, as demonstrated in [24]. As in the SS algorithm, the virtual array is divided into $N_{v}$ overlapping subarrays, each containing $N_{v}$ sensors. The $i$-th subarray has sensors located at $\left\{(1+n-i) d n=0,1, \ldots, N_{v}-1\right\}$ and is represented by

$$
\mathbf{z}_{1 i}=A_{1 i} \mathbf{p}+\sigma_{n}^{2} e_{i}
$$

where $A_{1 \mathrm{i}}$ is an $N_{v} \times \mathrm{K}$ matrix consisting of the $\left(N_{v}-i+1\right)$ th to $\left(2 N_{v}-i\right)^{\text {th }}$ rows of $A_{v}$ and $e_{i}$ is a vector consisting entirely of zeros except for 1 in the $i$-th position. From (5) and (10), we can obtain the smoothed covariance matrix as follows:

$$
\mathrm{R}_{\mathrm{ss}} \triangleq \frac{1}{N_{v}} \sum_{i=1}^{N_{v}} \mathbf{z}_{1 i} \mathbf{z}_{1 i}^{\mathbf{H}}=\frac{1}{N_{v}}\left(A_{11} \Lambda A_{11}^{\mathbf{H}}+\sigma_{n}^{2} \mathbf{I}\right),
$$

where $\Lambda=\operatorname{diag}\left(\sigma_{1}^{2}, \sigma_{2}^{2}, \ldots, \sigma_{K}^{2}\right)$.

As seen from (11), $\mathrm{R}_{\mathrm{ss}}$ can be treated as the covariance matrix of an $N$-element ULA whose array manifold is represented by $A_{11}$. Thus, many subspace-based DOA estimation methods, such as MUSIC, can be applied.

2.4. ES-DOA Algorithm. Compared to the classical MUSIC algorithm, the ES-DOA algorithm not only utilizes the noise subspace characteristics for DOA estimation but also makes use of the signal subspace characteristics.

The source covariance matrix is reconstructed into a Toeplitz matrix as follows:

$$
\mathbf{R}_{\mathbf{X}}=\mathbf{R}+J \mathbf{R}^{*} J
$$

where J denotes the $\mathrm{N} \times \mathrm{N}$ exchange matrix with ones on its antidiagonal and zeros elsewhere. The reconstructed matrix $\mathrm{R}_{x}$ can be decomposed as

$$
\mathbf{R}_{\mathbf{X}}=U_{s} \Lambda_{s} U_{S}^{H}+U_{U} \Lambda_{U} U_{U}^{H},
$$

where the subscripts $S$ and $U$ represent the signal subspace part and the noise subspace part, respectively.

The generalized inverse matrix $\mathbf{R}_{\mathbf{A}}^{+}$of the signal subspace part can be calculated as

$$
\mathbf{R}_{\mathbf{A}}^{+}=U_{s} \Lambda_{s}^{-1} U_{S}^{H} .
$$

The spatial frequency spectrum function of ES-DOA is defined as 


$$
P_{\mathrm{ES}}(\theta)=\frac{a^{H}\left(\theta_{i}\right) \mathbf{R}_{\mathrm{A}}^{+} a\left(\theta_{i}\right)}{a^{H}\left(\theta_{i}\right) U_{N} U_{N}^{H} a\left(\theta_{i}\right)} .
$$

The ES-DOA algorithm can achieve high resolution and demonstrates a strong antinoise capability when dealing with coherent array signals.

\section{Proposed UDC-DOA Method}

In this section, we will introduce the UDC-DOA method for addressing coherent signals received by a ULA.

Suppose that there are $K$ coherent signals impinging on an $N$-element ULA at different azimuths $\left\{\theta_{1}, \theta_{2}, \ldots, \theta_{K}\right\}$. Since coherent signals can be regarded as phase-delayed amplitude-weighted replicas of each other, the observed signal can be expressed as

$$
\mathbf{x}_{\mathrm{co}}(t)=\mathbf{A} \boldsymbol{\gamma} s_{1}(t)+\mathbf{n}(t),
$$

where $\gamma=\left[\gamma_{1}, \gamma_{2}, \ldots, \gamma_{K}\right] \in \mathbb{C}^{K}$ represents the complex attenuation vector of the coherent source signals with respect to the first signal $s_{1}(t)$. Suppose that $E\left(\left|s_{1}(t)\right|^{2}\right)=1$; then, using (3)-(5), the covariance matrix reduces to

$$
r_{m n}=\sum_{i=0}^{K} \sum_{j=0}^{K} \gamma_{i} \gamma_{j}^{*} e^{-j \pi\left[(m-1) \sin \left(\theta_{i}\right)-(n-1) \sin \left(\theta_{j}\right)\right]}+\sigma_{n}^{2} .
$$

It is clear that the signal covariance matrix $\mathbf{R}_{\mathrm{co}}$ is no longer nonsingular in this case. From (4) and (16), we can rewrite the covariance matrix as

$$
\widehat{\mathbf{R}}_{\mathrm{co}}=\frac{1}{M} \sum_{i=1}^{M} \mathbf{x}_{\mathrm{co}}\left(t_{i}\right) \mathbf{x}_{\mathrm{co}}\left(t_{i}\right)^{\mathbf{H}}=\left[\begin{array}{cccc}
r_{11} & r_{12} & \cdots & r_{1 N} \\
r_{21} & r_{22} & \cdots & r_{2 N} \\
\vdots & \vdots & & \vdots \\
r_{N 1} & r_{N 2} & \cdots & r_{N N}
\end{array}\right] .
$$

Inspired by the coarray-based estimators employed in cases of sparse arrays, we attempt to convert the sample covariance matrix of the signals observed by the ULA into the difference coarray domain. Since the product of $\gamma \gamma^{\mathrm{H}}$ is no longer diagonal in general in this case, we cannot vectorize $\mathbf{R}_{\mathrm{co}}$ by using Property 1 directly.

For convenience in obtaining the equivalent observed signal in the difference coarray mode for the ULA, we introduce the difference set $D$ as follows:

$$
D=\{i-j\}, \quad \forall i, j=1,2, \ldots, N .
$$

The difference value $d$ ranges from $1-N$ to $N-1$, and the weight function of the difference value can be expressed as

$$
\omega\left(d_{i j}\right)=N-|i-j|, \quad \forall i, j=1,2, \ldots, N .
$$

The difference coarray of the ULA can be constructed via the following steps:

(1) The covariance matrix $R_{c o}$ is rotated clockwise by $90^{\circ}$. As shown in Figure 1, each column corresponds to a distinct difference value, ranging from $(N-1)$ to $(1-N)$ when moving from left to right. Therefore, the elements in each column share the same difference value.

(2) By calculating the mean value of each column, the new observation value can be obtained as follows:

$$
z_{p}=\mathbf{f}\left(r_{i j}\right)=\frac{1}{\omega\left(d_{i j}\right)} \sum_{\substack{i-j=p-N \\ i, j \in[1, N]}} r_{i, j}, \quad p \in[1,(2 N-1)]
$$

(3) It is clear that the resulting difference coarray is equal to another longer, virtual ULA with $(2 N-1)$ elements. Following the process described above, the observation vector $\mathbf{z}$ of the virtual ULA can be expressed as

$$
\mathbf{z}=\mathscr{F}\left(\mathbf{R}_{\mathrm{co}}\right)=\mathbf{B q}+\sigma_{n}^{2} I,
$$

where the matrix $\mathbf{B} \in \mathbb{C}^{(2 N-1) \times K}$ represents the new array manifold of the virtual ULA, $\mathbf{q}$ is the equivalent source signal vector, and $\mathscr{F}$ represents the transition function of the matrix $R_{c o}$ that is mentioned above.

Then, we can obtain the covariance matrix of the new observed signals as follows:

$$
\mathrm{R}_{\mathrm{z}} \triangleq \mathrm{z}^{\mathrm{H}} \mathrm{Z}=\left[\mathrm{z}_{1}, \mathrm{z}_{2}, \ldots, \mathrm{z}_{2 N-1}\right]^{\mathrm{H}}\left[\mathrm{z}_{1}, \mathrm{z}_{2}, \ldots, \mathrm{z}_{2 N-1}\right] .
$$

Since the SS method can be used as a technique for building up the rank of an observation matrix for any array whose difference coarray is a filled ULA [24], this method can also naturally be applied to a ULA.

By applying the SS method, the virtual array is divided into $N$ overlapping subarrays, each containing $N$ sensors. According to (3) and (22), the covariance matrix of the $i$-th subarray can be expressed as

$$
R_{z i} \triangleq z_{i}^{\mathrm{H}} z_{i}=B_{1} \Phi^{i-1} \mathbf{q q}^{\mathbf{H}}\left(\Phi^{i-1}\right)^{\mathbf{H}} B_{1}^{\mathrm{H}}+\sigma_{n}^{2} \mathbf{I},
$$

where $B_{1}$ is the submatrix consisting of the first to $N$-th rows of $\mathbf{B}$. $\phi^{\mathrm{i}-1}$ denotes the $i$-th power of the $K \times K$ diagonal matrix

$$
\Phi=\operatorname{Diag}\left[e^{-j \pi \sin \left(\theta_{1}\right)}, e^{-j \pi \sin \left(\theta_{2}\right)}, \ldots, e^{-j \pi \sin \left(\theta_{K}\right)}\right] .
$$

From (5), (6), and (23), the spatially smoothed covariance matrix is defined as the mean of the forward-backward subarray covariance matrices

$$
\begin{aligned}
\mathbf{R}_{\mathrm{zss}} & \triangleq \frac{1}{N} \sum_{i=1}^{N} G_{i}\left(\mathbf{R}_{\mathrm{zz}}+\mathrm{JR}_{\mathrm{zz}}^{*} J\right) G_{i}^{\mathrm{T}}, \\
G_{i} & =\left[0_{N \times(i-1)}\left|\mathbf{I}_{N}\right| 0_{N \times(N-i)}\right],
\end{aligned}
$$

where $G_{i}$ represents the selection matrix for the $i$-th subarray.

Now, the DOA spectrum can be estimated using a general subspace-based algorithm such as MUSIC. In this paper, we prefer to use the ES-DOA algorithm to calculate 


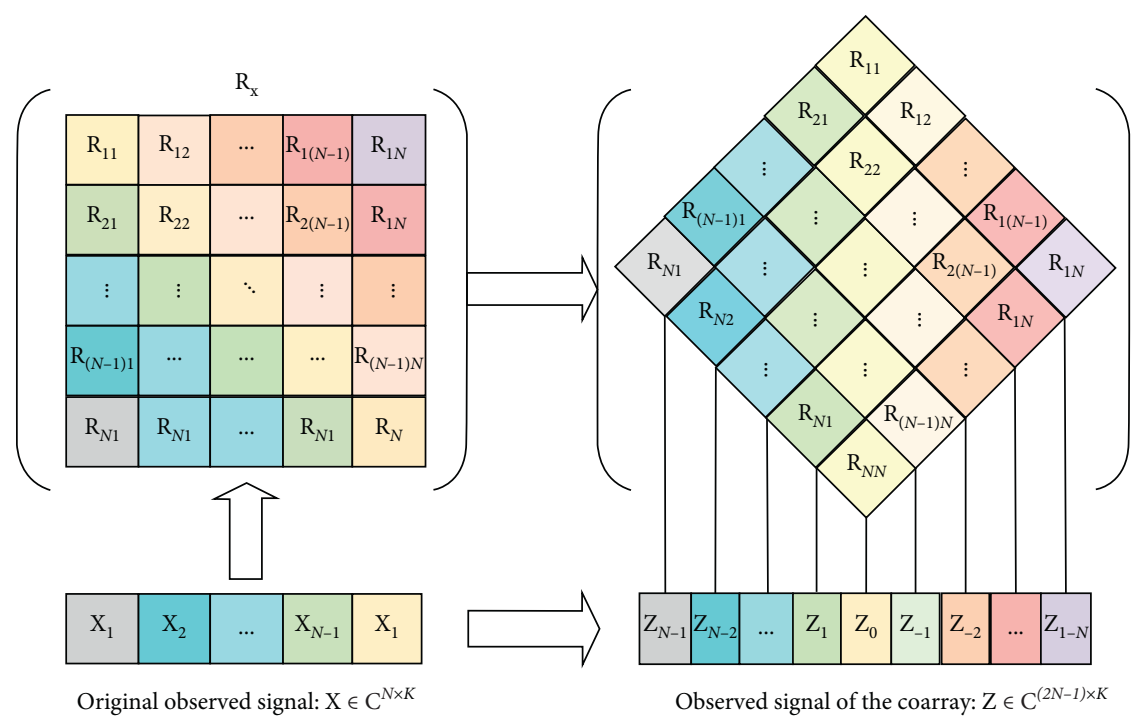

FIGURE 1: Illustration of the transformation of the observed signals from the original ULA to the virtual ULA based on the difference coarray.

the DOA spectrum because of its antinoise capability and robustness.

Notably, the original purpose of using SS method is to be able to exploit the DOFs of the difference coarray instead of decorrelating the coherent sources. In addition, the sparse arrays, such as nested arrays and coprime arrays, can commonly handle only uncorrelated signals. However, it is exciting and interesting that this restriction to uncorrelated signals no longer exists when the difference coarray is converted from a ULA.

As mentioned in Section 2, the ES-DOA algorithm can increase the rank of the source covariance matrix only by one in the coherent case, and it cannot be applied in the case of a sparse array. However, by virtue of the characteristics of ULAs, the ES-DOA algorithm can enhance the resolution and robustness performance in cases involving UDC signals.

Thus, our proposed method demonstrates improved accuracy and robustness in both the uncorrelated and coherent cases.

\section{Numerical Examples}

In this section, we employ numerical simulations to evaluate the validity of the proposed UDC-DOA method and compare its performance with that of conventional approaches. We assume that there are 11 far-field signals impinging on a ULA composed of 23 sensors. The locations of the signals range from $-50^{\circ}$ to $+50^{\circ}$, in increments of $10^{\circ}$. The spatial noise is zero-mean uniform white complex Gaussian noise. We simulate the DOA spectra under different SNR conditions and with different numbers of snapshots in both the uncorrelated case and coherent cases.

4.1. Uncorrelated Case. First, we evaluate the performance of the proposed method in the uncorrelated case. Suppose that all the incident signals are completely uncorrelated. The corresponding DOA spectra estimated using the classic MUSIC algorithm, the FBSS-MUSIC algorithm, and the UDC-based MUSIC (UDC-MUSIC) algorithm are compared under different SNR conditions in Figure 2 and under different snapshot conditions in Figure 3.

4.1.1. Different SNR Conditions. As seen in Figure 2(a), with 200 signal snapshots, the spectra peaks obtained with all three algorithms are in good agreement with the true DOAs under good noise conditions, that is, with an SNR of $10 \mathrm{~dB}$. As the SNR deteriorates to $0 \mathrm{~dB}$, small but obvious peak deviations occur in the spectrum of the FBSS-MUSIC method, as shown in Figure 2(b). In this case, the spectral peaks obtained using the proposed method become sharper than those of the classic MUSIC algorithm. As shown in Figure 2(c), as the SNR continues to decline to $-10 \mathrm{~dB}$, the spectrum of the FBSS-MUSIC algorithm significantly deteriorates, with the peaks deviating considerably from the true DOAs. Meanwhile, for the classic MUSIC algorithm, the peaks become blurred and inconspicuous while the spectral peaks found with the proposed algorithm remain accurate and sharp.

The results showed in Figure 2 indicate that the proposed method can achieve better resolution and robustness compared to the traditional algorithm, especially under severe noise interference conditions.

4.1.2. Different Number of Signal Snapshots. In Figure 3, the SNR condition is set to $5 \mathrm{~dB}$, and the DOA spectra in (a), (b), and (c) are calculated with different numbers of signal snapshots: 100, 20, and 5, respectively. By comparing the results, we find that the proposed method and FBSSMUSIC both demonstrate similar resolution performance that is superior to that of the classic MUSIC algorithm when the number of signal snapshots is sufficient. However, the FBSS-MUSIC algorithm is sensitive to the number of snapshots, causing the peaks to severely deviate from the 

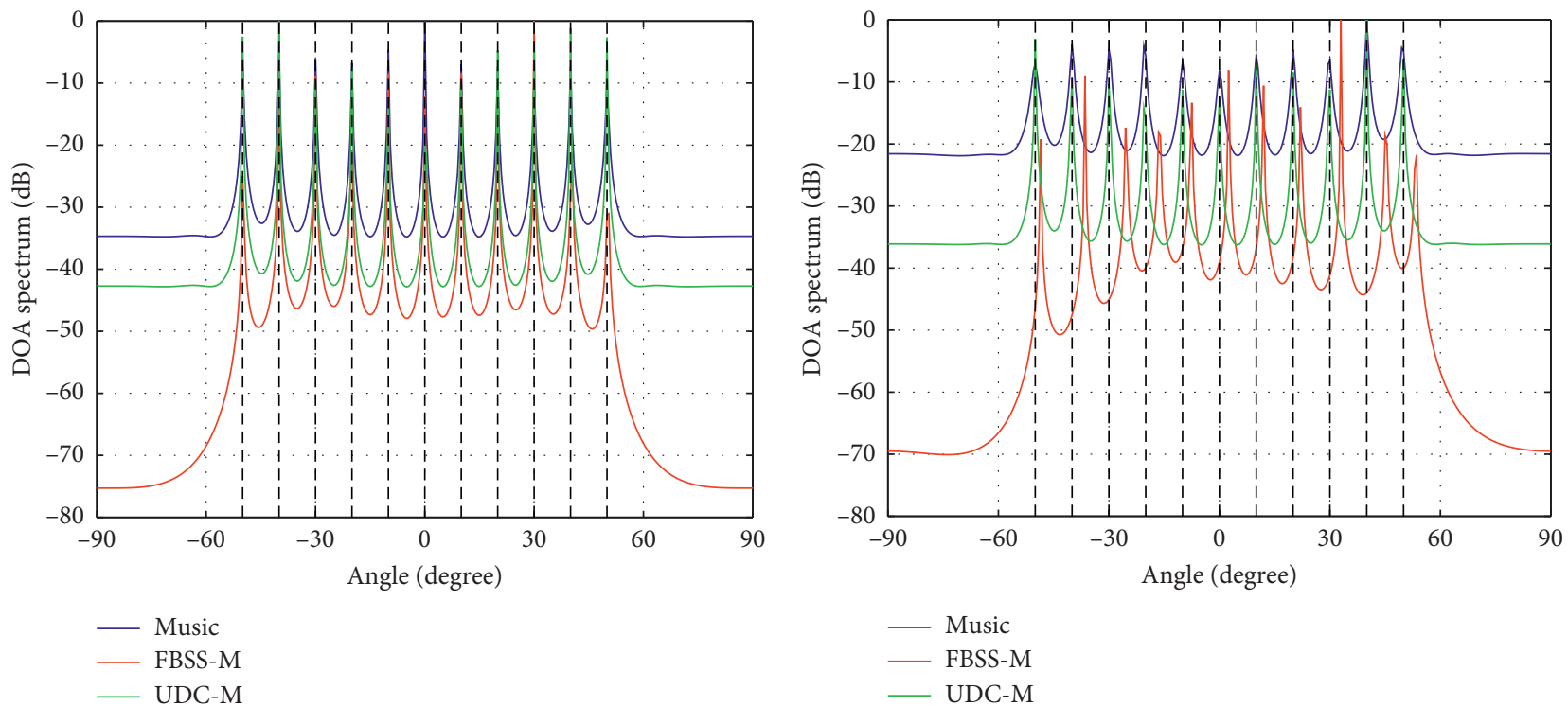

(a)

(b)

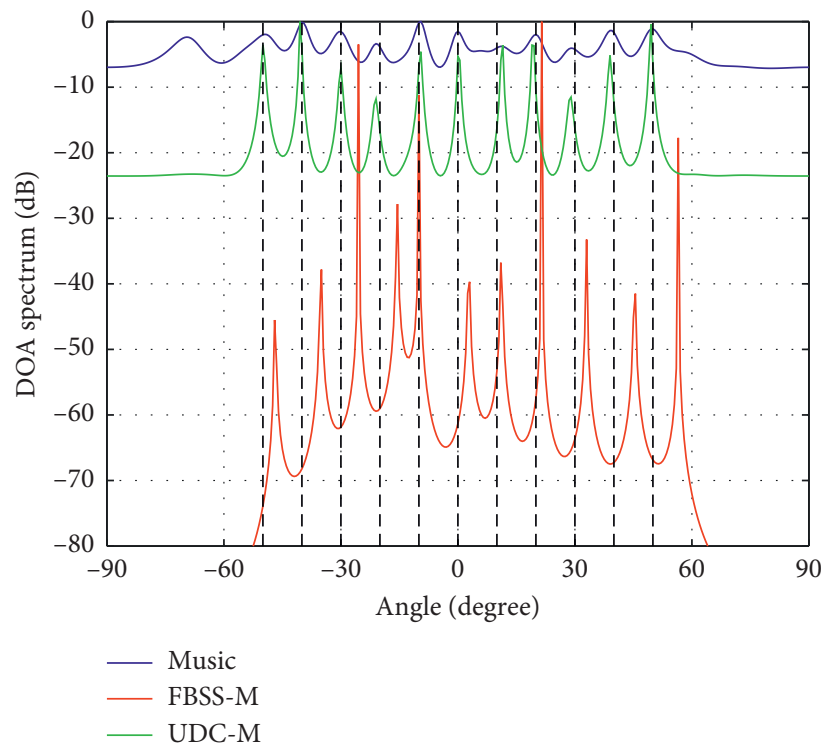

(c)

FIGURE 2: DOA spectra of the various algorithms for uncorrelated signals with 200 signal snapshots: (a) $\mathrm{SNR}=10 \mathrm{~dB},(\mathrm{~b}) \mathrm{SNR}=0 \mathrm{~dB}$, and (c) $\mathrm{SNR}=-10 \mathrm{~dB}$.

true values as the number of snapshots decreases. Moreover, although there are no obvious false peaks in the DOA spectrum of the classic MUSIC algorithm, the spectrum shows significant deterioration in resolution and even loses some peaks when the number of snapshots is very limited. In contrast, the proposed method shows reliable performance even with only a few signal snapshots. As shown in Figure 3(c), even with only five snapshots, the DOA peaks yielded by the proposed method remain accurate and sharp.
The results shown in Figure 3 indicate that the proposed method can maintain a high resolution and robustness even when there are only a few signal snapshots, while the traditional methods cannot function properly under these conditions.

4.2. Coherent Case. Second, we evaluate the performance of the proposed method in the coherent case. Suppose that all the incident signals are coherent, while the other simulation 


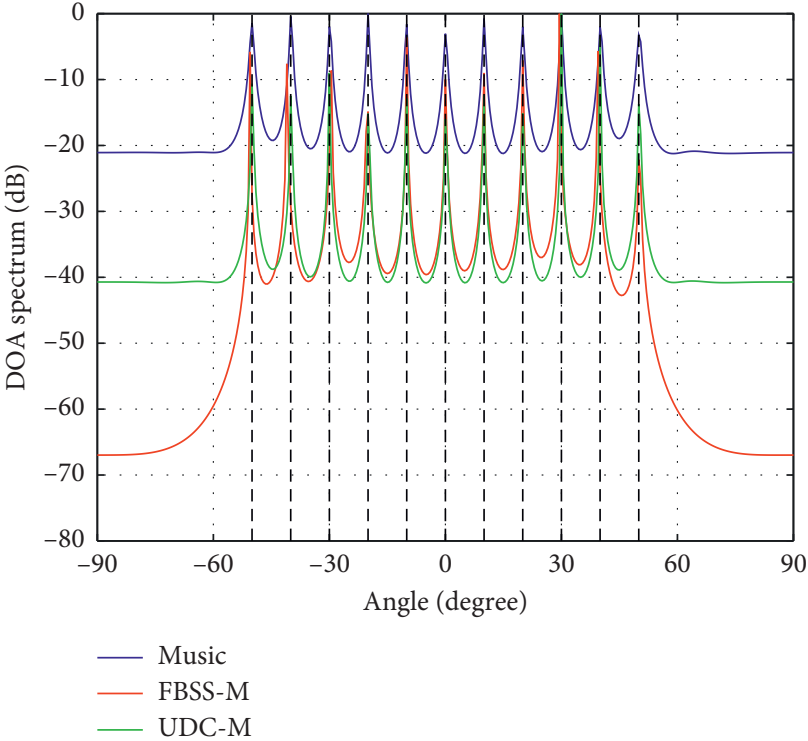

(a)

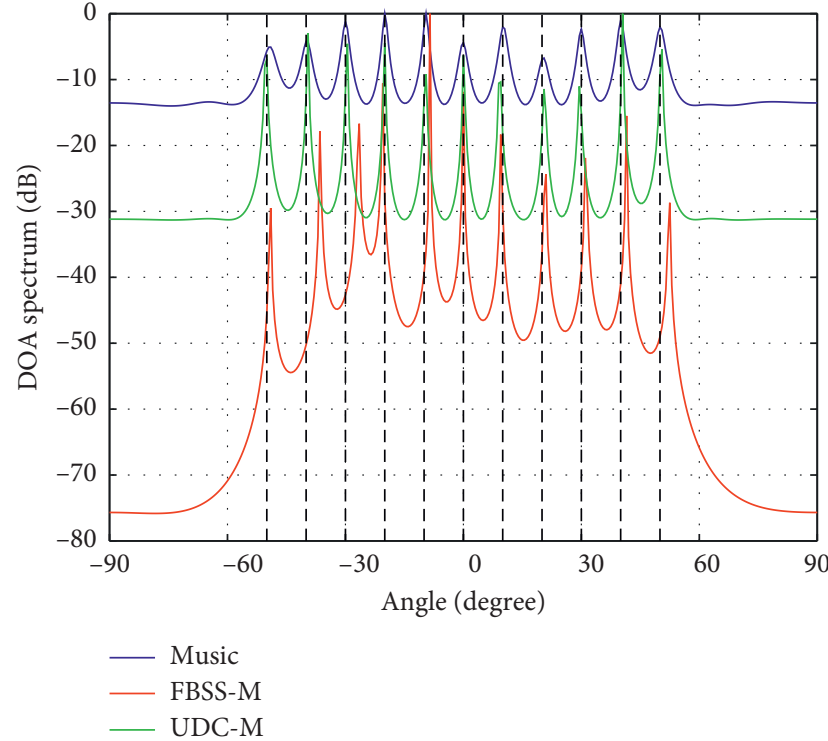

(b)

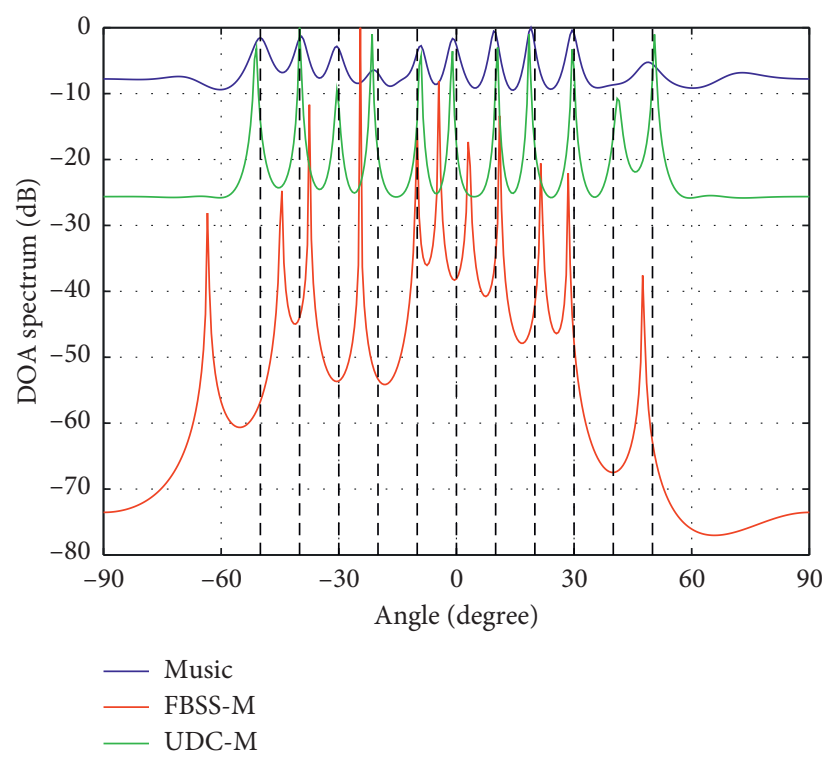

(c)

FIGURE 3: DOA spectra of the various algorithms for uncorrelated signals when SNR $=5 \mathrm{~dB}$ : (a) 100 signal snapshots, (b) 20 signal snapshots, and (c) 5 signal snapshots.

conditions are similar to the uncorrelated case. Since the classic MUSIC algorithm does not work in this case, we present only the performance comparison between the FBSS-MUSIC algorithm and the UDC-MUSIC algorithm.

4.2.1. Different SNR Conditions. As shown in Figure 4, the FBSS-MUSIC algorithm achieves better performance than the proposed algorithm under good signal conditions, such as $\mathrm{SNR}=10 \mathrm{~dB}$. However, the performance of the FBSSMUSIC algorithm significantly deteriorates with the degradation of the SNR. When the SNR reaches $-10 \mathrm{~dB}$, the peaks in the spectrum obtained with the FBSS-MUSIC algorithm severely deviate from the true DOAs, while the results of the proposed UDC-MUSIC algorithm are still acceptably accurate.

4.2.2. Different Signal Snapshots. As shown in Figure 5(a), with 100 signal snapshots, both the UDC-MUSIC method and the FBSS-MUSIC method show good accuracy performance, and the peaks obtained with the latter are even sharper. However, similar to the uncorrelated case, the DOA peaks in the FBSS-MUSIC spectrum rapidly deviate from the true values as the number of snapshots decreases, while the proposed method shows a superior capability to cope with very few snapshots. 


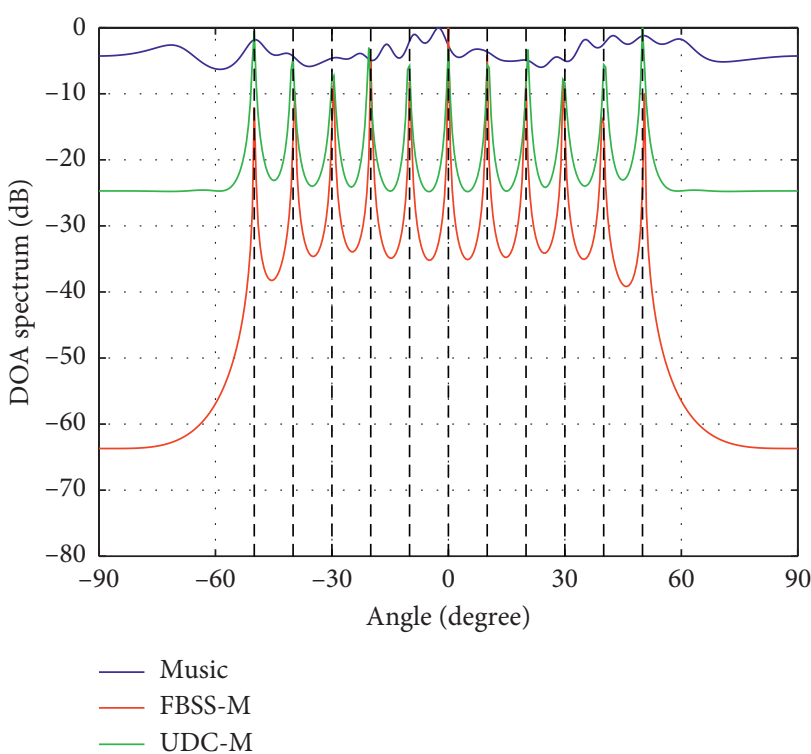

(a)

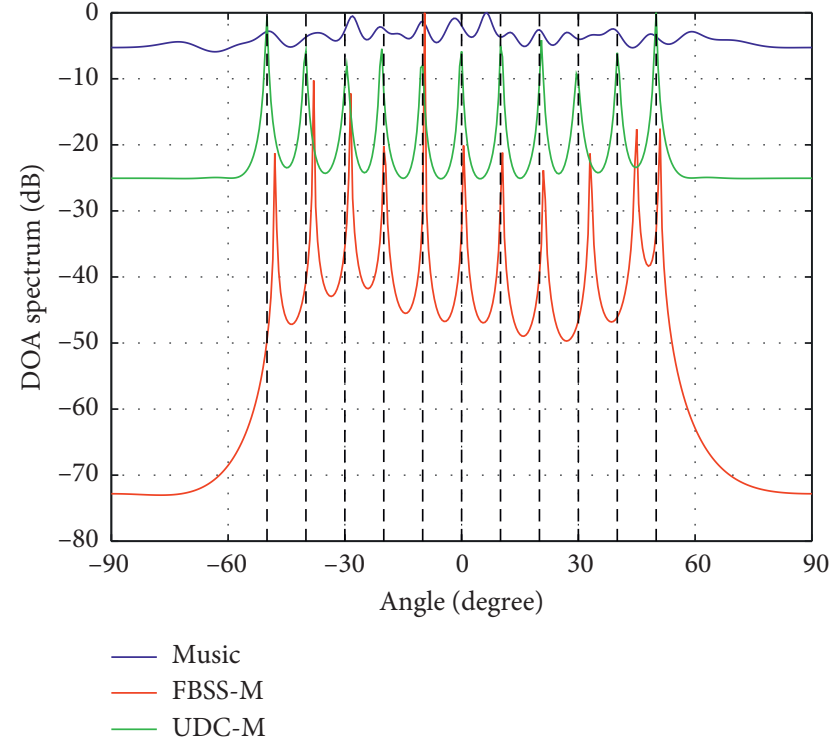

(b)

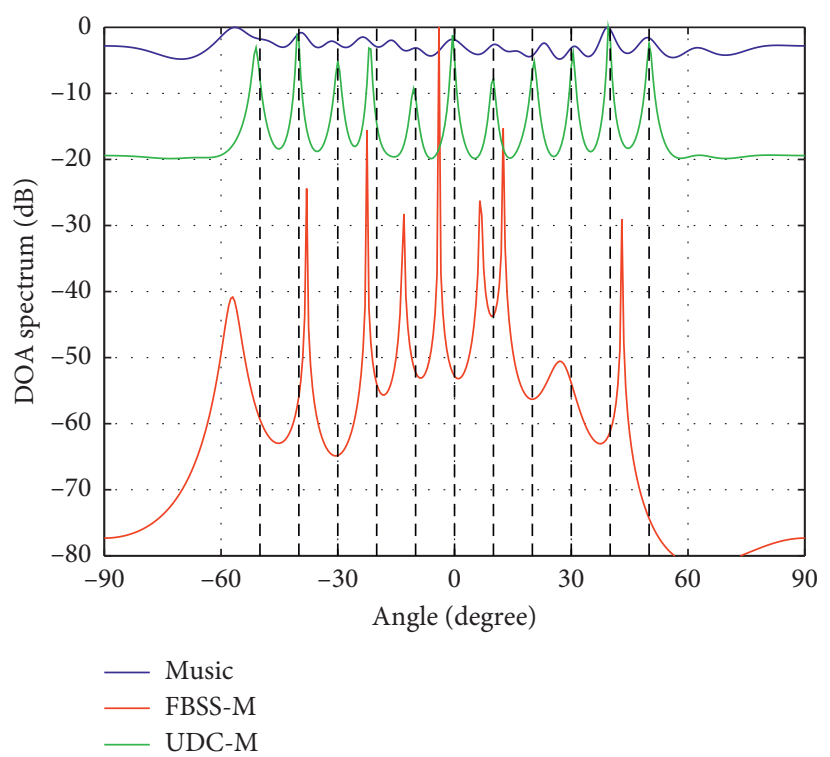

(c)

FiguRE 4: DOA spectra of the various algorithms for coherent signals with 200 signal snapshots: (a) $\mathrm{SNR}=10 \mathrm{~dB},(\mathrm{~b}) \mathrm{SNR}=0 \mathrm{~dB}$, and (c) $\mathrm{SNR}=-10 \mathrm{~dB}$.

4.3. UDC-Based ES-DOA Algorithm. In the previous simulations, we have used the classic MUSIC algorithm to address the signals from the UDC. In this section, we evaluate the DOA estimation performance of the UDCbased ES-DOA (UDC-ES-DOA) method in both the uncorrelated and coherent cases.

As mentioned in Section 2, the ES-DOA algorithm can achieve a high resolution and has a strong antinoise capability. Thus, the following simulations correspond to challenging conditions, with the SNR set to $10 \mathrm{~dB}$ and only 50 snapshots.

Figure 6 shows the results for the uncorrelated case, while Figure 7 shows the results for the coherent case. In both figures, the UDC-ES-DOA method is denoted by UDC-
ES. It is clear that the DOA spectrum obtained with UDCES-DOA has the highest resolution in both cases. Although the UDC-MUSIC algorithm shows much better performance than the classic MUSIC and FBSS-MUSIC algorithms, we can still observe some blurred and inconspicuous peaks in its spectra. Meanwhile, the UDC-ES-DOA method identifies the DOAs of all signals with little deviation.

4.4. RMSE Evaluation via Monte Carlo Simulations. To verify the advantages of our proposed method, without loss of generality, we report Monte Carlo simulations carried out to evaluate the average root-mean-square error (RMSE) performance for DOA estimation and compare the results 

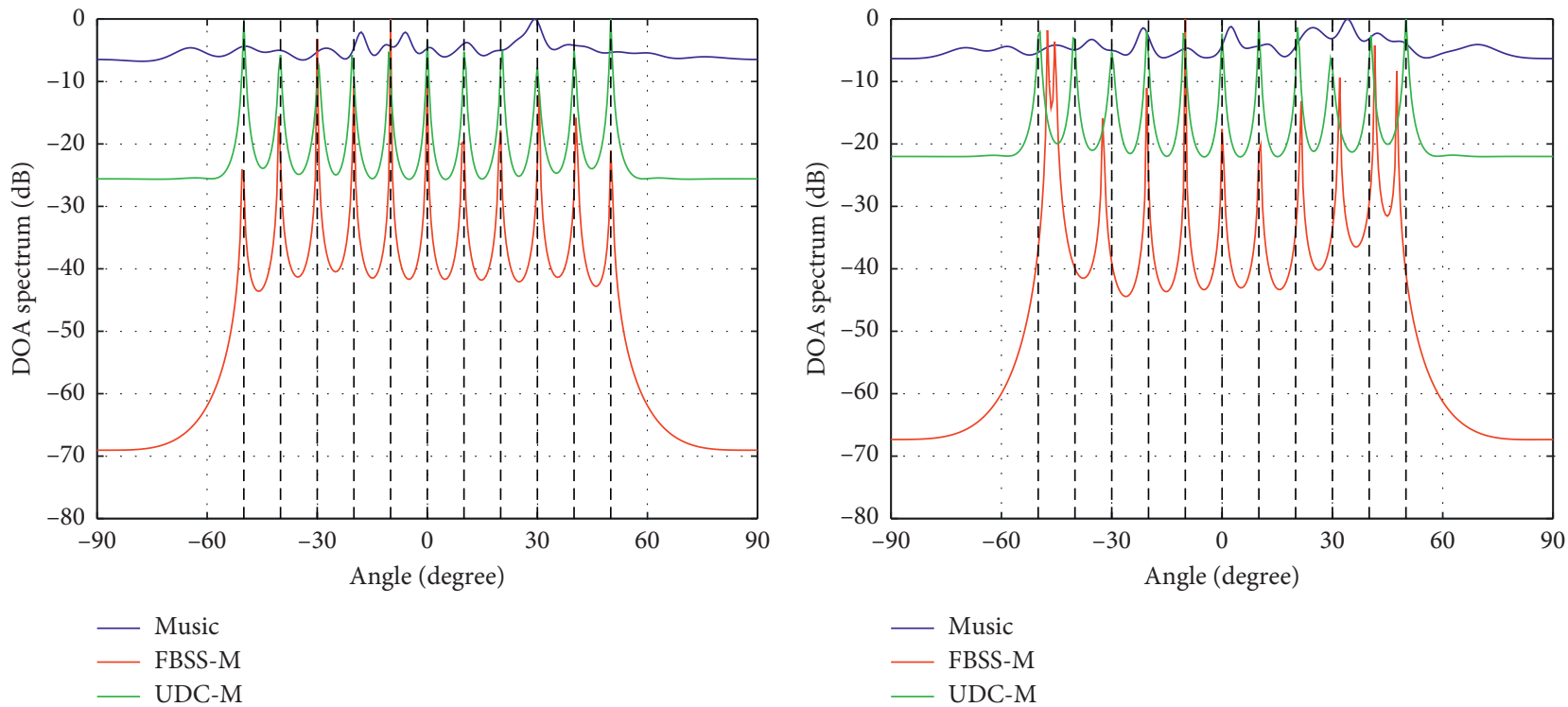

(a)

(b)

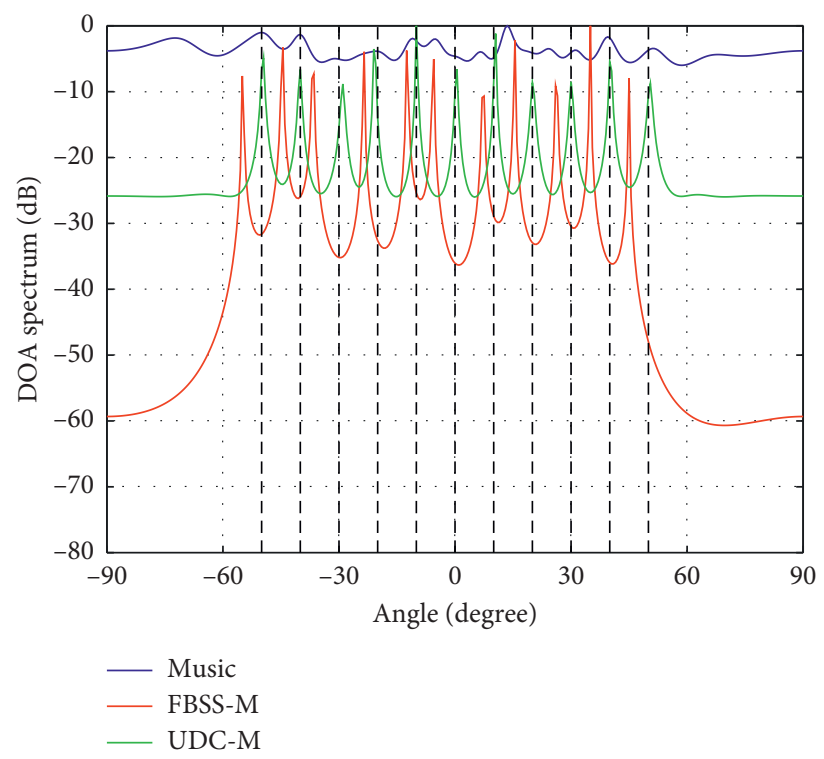

(c)

FIGURE 5: DOA spectra of the various algorithms for coherent signals when SNR $=5 \mathrm{~dB}$ : (a) 100 signal snapshots, (b) 20 signal snapshots, and (c) 5 signal snapshots.

with those of the other algorithms. The ULA topology and the incident signals remain the same as in the previous simulations.

We evaluate the RMSEs of the proposed method in both the uncorrelated and coherent cases. In both cases, the results are based on 200 independent Monte Carlo trials implemented to obtain the RMSE curves under different SNR and snapshot conditions. In the uncorrelated case, we compare the RMSE performances of the proposed UDC-ESDOA and UDC-MUSIC algorithms, the FBSS-MUSIC algorithm, and the classic MUSIC algorithm. In the coherent case, we compare the RMSE performances of the proposed UDC-ES-DOA and UDC-MUSIC algorithms, the FBSSMUSIC algorithm, and the $l_{1}$-SVD algorithm. The $l_{1}$-SVD algorithm is a kind of CS-based DOA approach which utilizes the $l_{1}$ norm minimization techniques by assuming the incoming DOAs specified on the discrete frequency grid [5]. In our simulation, the grid ranges from $-90^{\circ}$ to $90^{\circ}$ with the width of one degree.

Figure 8 depicts the RMSE curves with respect to the SNR when the number of snapshots is 200. From Figure 8(a), we find that the UDC-ES-DOA and UDC-MUSIC methods are obviously superior and that the UDC-ES-DOA method is significantly more robust when the SNR is below $-5 \mathrm{~dB}$. In contrast, the classic MUSIC algorithm is too sensitive to noise interference. The results for the coherent case are shown in Figure 8(b). Similar to the uncorrelated case, the UDC-ES-DOA and UDC-MUSIC methods perform much 


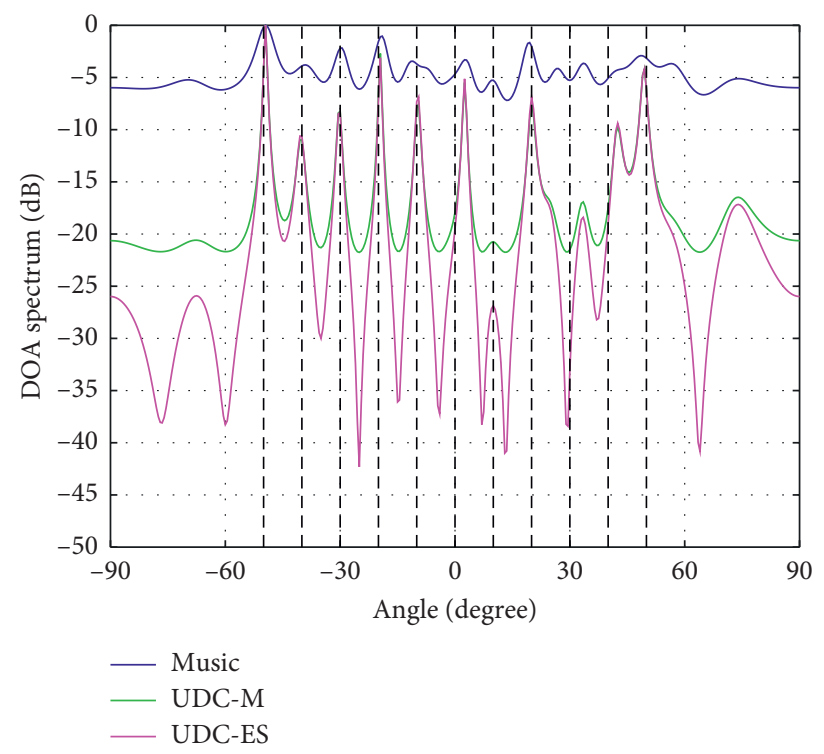

FIGURE 6: DOA spectrum of the various algorithms with uncorrelated signals with 50 signal snapshots when $\mathrm{SNR}=-10 \mathrm{~dB}$.

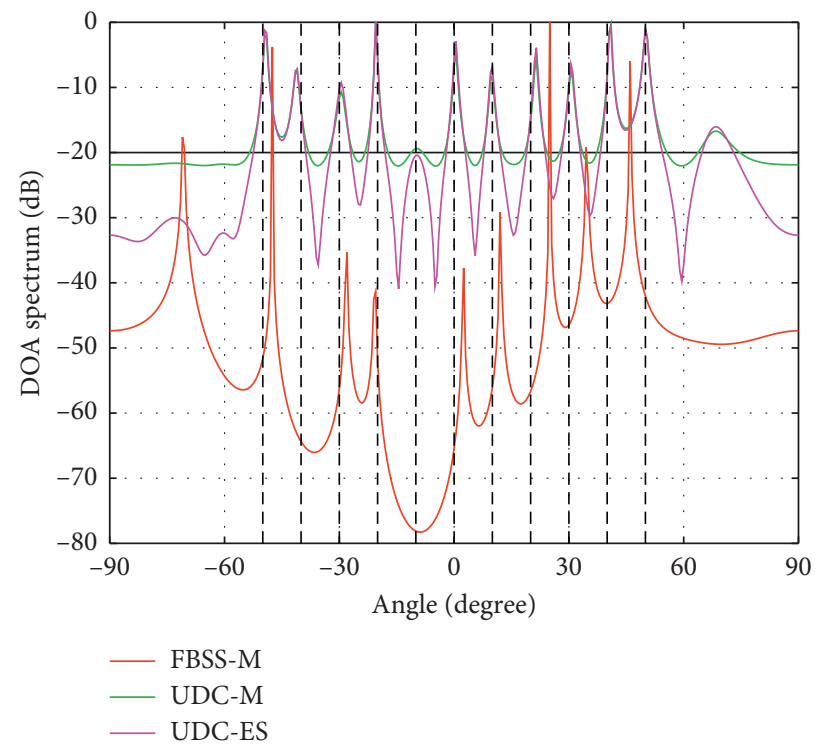

FIGURE 7: DOA spectrum of the various algorithms with coherent signals with 50 signal snapshots when $\mathrm{SNR}=-10 \mathrm{~dB}$.

better than the FBSS method within the low-SNR range. Although the $l_{1}$-SVD method is extremely reliable when the SNR is below $-10 \mathrm{~dB}$, it is surpassed by the proposed UDCES-DOA and UDC-MUSIC methods at the crossing points that occur at $-7 \mathrm{~dB}$ and $-5 \mathrm{~dB}$, respectively.

Figure 9 depicts the RMSE curves with respect to the number of snapshots when the SNR is $5 \mathrm{~dB}$. From Figure 9(a), we find that the UDC-ES-DOA and UDCMUSIC methods demonstrate significant superiority when the snapshots are limited, and the UDC-ES-DOA method performs more impressively with only a few snapshots while the classic MUSIC algorithm cannot estimate correctly. It can be seen that the above three algorithms have similar accuracy performances when the number of snapshots is more than 30. In contrast, the RMSE curve of the FBSS-
MUSIC algorithm indicates that it needs many more snapshots than the other three algorithms to achieve the same accuracy performance. The results for the coherent case are shown in Figure 9(b). Similar to the uncorrelated case, the UDC-ES-DOA and UDC-MUSIC methods perform much better than the FBSS method. Although the $l_{1}$ SVD method is extremely reliable when the SNR is below $-10 \mathrm{~dB}$, it is surpassed by the proposed UDC-ES-DOA and UDC-MUSIC methods at the crossing points of $-7 \mathrm{~dB}$ and $-5 \mathrm{~dB}$, respectively.

4.5. Computational Complexity. We evaluate the computational complexity of the proposed method and compare the results with those of the other methods. For a 


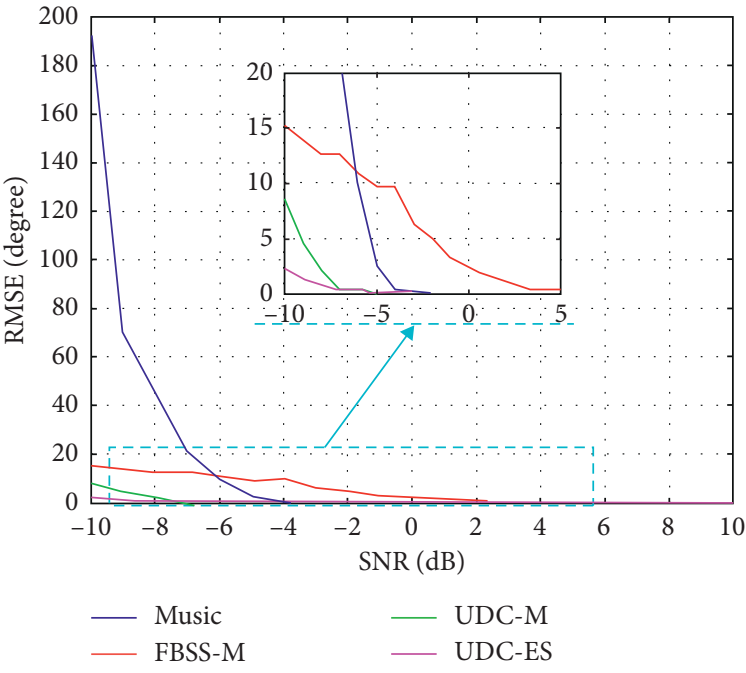

(a)

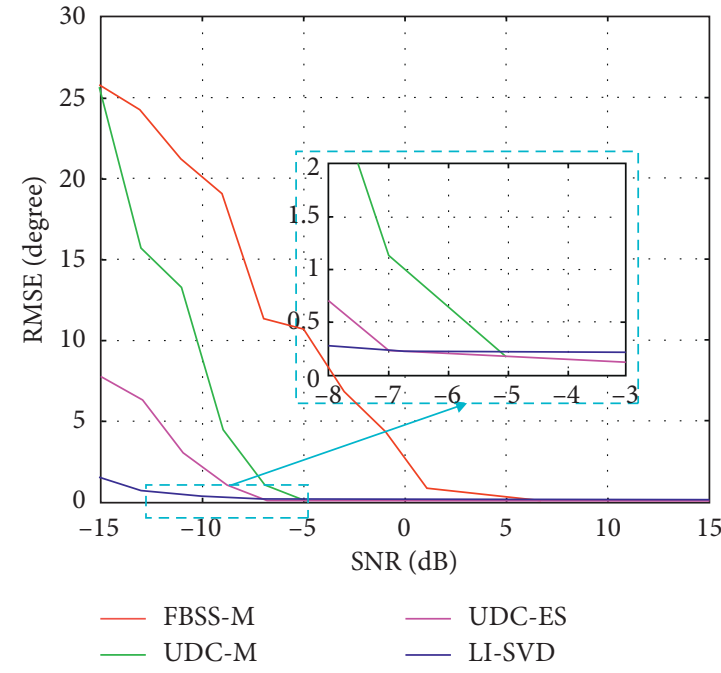

(b)

FIGURE 8: RMSE curves versus the SNR with 200 snapshots: (a) in the uncorrelated case and (b) in the coherent case.

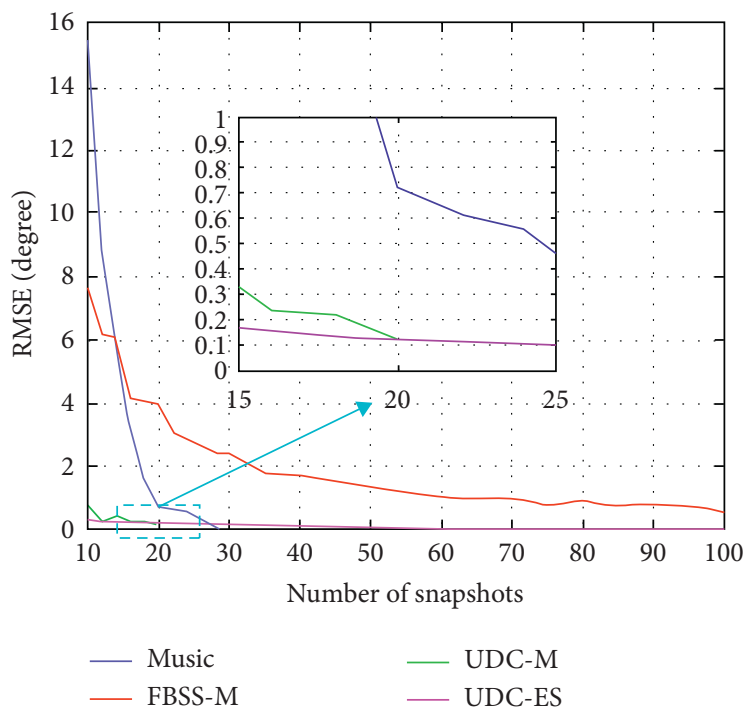

(a)

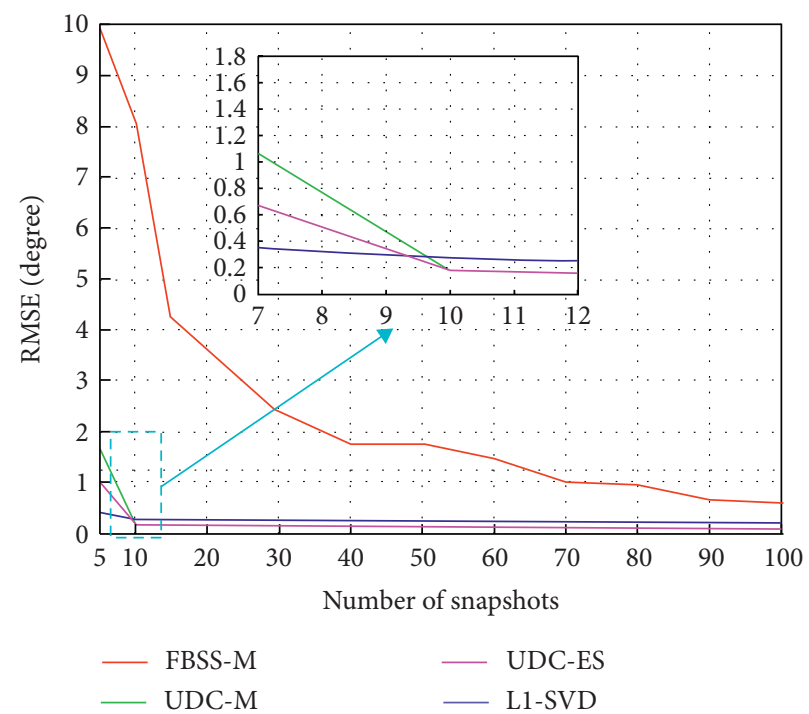

(b)

FIGURE 9: RMSE curves versus the number of snapshots when SNR $=5 \mathrm{~dB}$ : (a) in the uncorrelated case and (b) in the coherent condition.

quantitative comparison, the CPU running time (RT) of different algorithms is computed with the same data in both the uncorrelated and coherent cases. The CPU type is Intel Core i7-4790@3.6 GHz and we implemented the simulation in the MATLAB environment. We fix $K=11$, $N=23$, and $\mathrm{SNR}=5 \mathrm{~dB}$. And the simulation parameters and running time results are tabulated in Table 1.

It can be seen from the results that the computational complexity of the proposed method is slightly higher than the classic MUSIC and the FBSS method, while the CS-based algorithm takes several hundred times longer.
4.6. Remarks. From the simulation results above, it can be concluded that the proposed UDC-based methods are effective and impressive under both uncorrelated and coherent conditions. Compared to the traditional methods, the greatest advantage of the UDC-based methods is their outstanding robustness in cases of noise interference and a limited number of snapshots. Meanwhile, in the coherent source cases, the UDC-based methods can achieve similar DOA performance as the CS-based methods with significantly less computational complexity. According to the above evaluation and comparison based on Monte Carlo 
TABLE 1: CPU running time of each algorithm.

\begin{tabular}{lcccc}
\hline \multirow{2}{*}{ DOA method } & \multicolumn{2}{c}{ RT $(\mathrm{s})^{1}$} & \multicolumn{2}{c}{ RT (s) ${ }^{2}$} \\
& $L=20$ & $L=500$ & $L=20$ & $L=500$ \\
\hline MUSIC & 0.004 & 0.006 & - & - \\
FBSS-MUSIC & 0.007 & 0.008 & 0.007 & 0.008 \\
UDC-MUSIC & 0.010 & 0.012 & 0.010 & 0.012 \\
UDC-ES-DOA & 0.012 & 0.013 & 0.012 & 0.013 \\
l $_{1}$-SVD & - & - & 3.30 & 3.91 \\
\hline
\end{tabular}

${ }^{1} \mathrm{CPU}$ running time in the uncorrelated case; ${ }^{2} \mathrm{CPU}$ running time in the coherent signal source case.

trials, it is fair to state that the UDC-based approach constitutes a promising and attractive method that is especially suitable for low-SNR or snapshot-limited practical applications.

\section{Conclusions}

In this paper, we have introduced a difference-coarraybased (UDC-based) DOA method to address the problem of DOA estimation for coherent signals received by a ULA. Inspired by the coarray-based estimators employed in cases of sparse arrays, we convert the sample covariance matrix of the signals observed by the ULA into the difference coarray domain and process the signals with an SS method. The proposed method exhibits good accuracy and robustness in both the uncorrelated and coherent cases. Numerical simulations verify that the UDC-based method can achieve significantly improved DOA estimation accuracy even when the SNR is very low. In addition, the UDC-based method is insensitive to the number of snapshots and thus can achieve high accuracy with a limited number of snapshots. Under extremely challenging conditions, the UDC-ES-DOA method is preferred because of its robustness, while the UDC-MUSIC method is suitable for most moderate cases of lower complexity. Due to its demonstrated advantages, the UDC-based method is a promising and competitive approach for practical DOA estimation, especially for low-SNR or snapshot-limited applications.

\section{Data Availability}

The data used to support the findings of this study are available from the corresponding author upon request.

\section{Conflicts of Interest}

The authors declare that there are no conflicts of interest regarding the publication of this paper.

\section{Acknowledgments}

This work was supported by the National Science Foundation of China under Grant no. 61801497.

\section{References}

[1] H. Krim and M. Viberg, "Two decades of array signal processing research: the parametric approach," IEEE Signal Processing Magazine, vol. 13, no. 4, pp. 67-94, 1996.

[2] R. Schmidt, "Multiple emitter location and signal parameter estimation," IEEE Transactions on Antennas and Propagation, vol. 34, no. 3, pp. 276-280, 1986.

[3] R. Roy and T. Kailath, "Esprit-estimation of signal parameters via rotational invariance techniques," IEEE Transactions on Acoustics, Speech, and Signal Processing, vol. 37, no. 7, pp. 984-995, 1989.

[4] T.-J. Shan, M. Wax, and T. Kailath, "On spatial smoothing for direction-of-arrival estimation of coherent signals," IEEE Transactions on Acoustics, Speech, and Signal Processing, vol. 33, no. 4, pp. 806-811, 1985.

[5] S. U. Pillai and B. H. Kwon, "Forward/backward spatial smoothing techniques for coherent signal identification," IEEE Transactions on Acoustics, Speech, and Signal Processing, vol. 37, no. 1, pp. 8-15, 1989.

[6] R. T. Williams, S. Prasad, A. K. Mahalanabis, and L. H. Sibul, "An improved spatial smoothing technique for bearing estimation in a multipath environment," IEEE Transactions on Acoustics, Speech, and Signal Processing, vol. 36, no. 4, pp. 425-432, 1988.

[7] J. E. Dzielski, R. C. Burkhardt, and M. E. Kotanchek, "Comments on "modified MUSIC algorithm for estimating DOA of signals"” Signal Processing, vol. 55, no. 2, pp. 253-254, 1996.

[8] Z. Xiaofei, L. Wen, S. Ying, Z. Ruina, and X. Dazhuan, "A novel DOA estimation algorithm based on eigen space," in Proceedings of the 2007 International Symposium on Microwave, Antenna, Propagation and EMC Technologies for Wireless Communications, pp. 551-554, Hangzhou, China, August 2007.

[9] F.-M. Han and X.-D. Zhang, "An ESPRIT-like algorithm for coherent DOA estimation," IEEE Antennas and Wireless Propagation Letters, vol. 4, pp. 443-446, 2005.

[10] C. Li, G. Liao, S. Zhu, and S. Wu, “An ESPRIT-like algorithm for coherent DOA estimation based on data matrix decomposition in MIMO radar," Signal Processing, vol. 91, no. 8, pp. 1803-1811, 2011.

[11] S. Ren, X. Ma, S. Yan, and C. Hao, "2-D unitary ESPRIT-like direction-of-arrival (DOA) estimation for coherent signals with a uniform rectangular array," Sensors, vol. 13, no. 4, pp. 4272-4288, 2013.

[12] J. Xin and A. Sano, "MSE-based regularization approach to direction estimation of coherent narrowband signals using linear prediction," IEEE Transactions on Signal Processing, vol. 49, no. 11, pp. 2481-2497, 2001.

[13] M. Inoue, K. Hayashi, H. Mori, and T. Nabetani, "A DOA estimation method with kronecker subspace for coherent signals," IEEE Communications Letters, vol. 22, no. 11, pp. 2306-2309, 2018.

[14] L. Gan and X. Luo, "Direction-of-arrival estimation for uncorrelated and coherent signals in the presence of multipath propagation," IET Microwaves, Antennas \& Propagation, vol. 7, no. 9, pp. 746-753, 2013.

[15] Z. Ye, Y. Zhang, and C. Liu, "Direction-of-arrival estimation for uncorrelated and coherent signals with fewer sensors," IET Microwaves, Antennas \& Propagation, vol. 3, no. 3, pp. 473482, 2009.

[16] H. Shi, W. Leng, A. Wang, and T. Guo, "DOA estimation for mixed uncorrelated and coherent sources in multipath 
environment," International Journal of Antennas and Propagation, vol. 2015, Article ID 636545, 8 pages, 2015.

[17] D. Malioutov, M. Cetin, and A. S. Willsky, "A sparse signal reconstruction perspective for source localization with sensor arrays," IEEE Transactions on Signal Processing, vol. 53, no. 8, pp. 3010-3022, 2005.

[18] M. M. Hyder and K. Mahata, "Direction-of-arrival estimation using a mixed $l_{2,0}$ norm approximation," IEEE Transactions on Signal Processing, vol. 58, no. 9, pp. 4646-4655, 2010.

[19] Z.-M. Liu and Y.-Y. Zhou, "A unified framework and sparse bayesian perspective for direction-of-arrival estimation in the presence of array imperfections," IEEE Transactions on Signal Processing, vol. 61, no. 15, pp. 3786-3798, 2013.

[20] P. Pal and P. P. Vaidyanathan, "Pushing the limits of sparse support recovery using correlation information," IEEE Transactions on Signal Processing, vol. 63, no. 3, pp. 711-726, 2015.

[21] P. Pal and P. P. Vaidyanathan, "Correlation-aware techniques for sparse support re-covery," in Proceedings of the IEEE Statistical Signal Processing Workshop (SSP), pp. 53-56, Ann Arbor, MI, USA, August 2012.

[22] C. Zhou, Y. Gu, Y. D. Zhang, Z. Shi, T. Jin, and X. Wu, "Compressive sensing-based coprime array direction-of-arrival estimation," IET Communications, vol. 11, no. 11, pp. 1719-1724, 2017.

[23] P. P. Vaidyanathan and P. Pal, "Sparse sensing with co-prime samplers and arrays," IEEE Transactions on Signal Processing, vol. 59, no. 2, pp. 573-586, 2011.

[24] P. Pal and P. P. Vaidyanathan, "Nested arrays: a novel approach to array processing with enhanced degrees of freedom," IEEE Transactions on Signal Processing, vol. 58, no. 8, pp. 4167-4181, 2010.

[25] K. Han and A. Nehorai, "Improved source number detection and direction estimation with nested arrays and ULAs using jackknifing," IEEE Transactions on Signal Processing, vol. 61, no. 23, pp. 6118-6128, 2013.

[26] M. Yang, L. Sun, X. Yuan, and B. Chen, "Improved nested array with hole-free DCA and more degrees of freedom," Electronics Letters, vol. 52, no. 25, pp. 2068-2070, 2016.

[27] J. Liu, Y. Zhang, Y. Lu, S. Ren, and S. Cao, "Augmented nested arrays with enhanced dof and reduced mutual coupling," IEEE Transactions on Signal Processing, vol. 65, no. 21, pp. 5549-5563, 2017.

[28] Z. Zheng, W.-Q. Wang, Y. Kong, and Y. D. Zhang, "MISC array: a new sparse array design achieving increased degrees of freedom and reduced mutual coupling effect," IEEE Transactions on Signal Processing, vol. 67, no. 7, pp. 1728-1741, 2019.

[29] M. Wang and A. Nehorai, "Coarrays, MUSIC, and the cramér-rao bound," IEEE Transactions on Signal Processing, vol. 65, no. 4, pp. 933-946, 2017.

[30] C.-L. Liu and P. P. Vaidyanathan, "Remarks on the spatial smoothing step in coarray MUSIC," IEEE Signal Processing Letters, vol. 22, no. 9, pp. 1438-1442, 2015.

[31] W.-K. Ma, T.-H. Hsieh, and C.-Y. Chi, "DOA estimation of quasi-stationary signals with less sensors than sources and unknown spatial noise covariance: a khatri-rao subspace approach," IEEE Transactions on Signal Processing, vol. 58, no. 4, pp. 2168-2180, 2010. 